\title{
A comparative study to find the effectiveness of weight bearing exercises on stable platform versus wobble board, to improve balance and functional outcome of individuals with knee osteoarthritis
}

\author{
K. Kotteeswaran', P. Sindhura ${ }^{2}$, P. Mohith Sesikiran², Vengata Subramani Manoharan ${ }^{3}$ \\ ${ }^{1}$ Professor, ${ }^{2}$ Post Graduate, Saveetha College of Physiotherapy, Saveetha Institute of Medical and Technical Sciences, \\ Chennai, India \\ ${ }^{3}$ Lecturer, Physiotherapy Department, University of Kuala Lumpur Royal College of Medicine Perak, Malaysia
}

(Received: April $2020 \quad$ Revised: July $2020 \quad$ Accepted: August 2020)

Corresponding author: K. Kotteeswaran. Email: k.kotteeswaran@gmail.com

\begin{abstract}
Introduction and Aim: Knee osteoarthritis is a common degenerative disease, with its prevalence increasing with age and with multifactorial aetiology. It is a common cause of disability worldwide. Aim of the study is to compare the effect of weight bearing exercises on wobble board and stable platform in improving balance and functional outcome by the pre and post analysis.

Materials and Methods: Experimental study was conducted in Saveetha hospital, Chennai. Forty subjects including both males and females diagnosed with osteoarthritis knee within the age of 40 to 70 years were recruited and divided into two groups with 20 subjects in each group (Group A- 20 subjects, Group B- 20 subjects). Group A was given weight bearing exercises on wobble board along with interferential therapy, in 4 sets with 6 repetitions in each set and a rest interval of one minute between each set, 15 minutes per session, 3 days/week for 4 weeks and Group B was given weight bearing exercises on stable platform along with interferential therapy in 4 sets with 6 repetitions in each set and a rest interval of one minute between each set, 15 minutes per session, 3 days/week for 4 weeks.
\end{abstract}

Results: At the end of the study, data was analysed and there was improvement in balance and functional outcome.

Conclusion: This study concluded that patients with knee osteoarthritis had improvement in balance and functional outcome through weight bearing exercises given on wobble board along with interferential therapy compared to the patients who were given weight-bearing exercises on stable platform along with interferential therapy.

Keywords: Osteoarthritis; wobble board; stable platform; interferential therapy.

\section{INTRODUCTION}

$\mathrm{O}$ steoarthritis is defined as a mixed group of conditions that lead to joint symptoms and signs that are associated with faulty integrity of articular cartilage and related changes in the underlying bone at the joint margins. Osteoarthritis is also called as a degenerative arthritis or osteoarthrosis (1). Prevalence of knee osteoarthritis increases with increase in age and it is one of the most common musculoskeletal disorder worldwide, affecting $30-40 \%$ of the population by the age of 65 years. $(2,3)$. Women are more affected by OA knee than men(4). Osteoarthritis is characterised by damageto the articular cartilage, osteophyte formation at the bone margins, sub chondral sclerosisand morphological changes of the synovial membrane and joint capsule (5).

Osteoarthritis have multifactorial aetiology, of which some of the causes include the popular squatting position, rising obesity, sedentary lifestyle, and poor diet $(5,6)$. Osteoarthritis is characterized by loss of normal configuration, crackling during movement, bone deformities, formation of bone spurs, the presence of inflammatory process, the accumulation of synovial fluid, weakness of quadriceps muscles and sensorimotor loss. Symptoms of knee osteoarthritis include joint pain particularly after prolonged activity and weight bearing, loss of motion, joint stiffness that is experienced after inactivity, decreased muscle strength, proprioceptive deficits, reduced balance, occasional swelling and crepitus $(1,7,8)$. Subjects with knee osteoarthritis show quadriceps muscle weakness as well as proprioceptive deficits, which can alter balance and postural control. Joint inflammation present in these patients contributes to pain and prevents the arrival of afferent information regarding movement and joint position. Such deficits cause a change in dynamic stability provided by muscles around the joint, generating a functional instability that limits the individual's ability to perform ADL's $(4,9)$.

The advantages of weight bearing exercises, strength training, co- ordination training, gait training is becoming more accepted and used in clinical practice and are found to improve balance $(2,10)$. Weight bearing exercises of lower extremity are performed with feet fixed on an object i.e., stable platform or unstable surface. Giving exercises in standing or weight bearing positions to individuals with painful osteoarthritis knee may aggravate symptoms like pain, swelling etc., $(2,11)$. However, conventionally IFT, TENS, ultrasound therapy and combination of 
these modalities have been used to reduce pain (1214) and strength training and balance training are usually performed in standing or weight bearing (2, 15).

Balance is a defined as the dynamics of body posture to prevent falling and it is related to the forces acting on the body and the characteristics of body segments (16). People with osteoarthritis knee sometimes report episodes of knee instability that may result in fall or limit their activities of daily living (17). Balance training is defined as exercises in which participants exercise their muscles against an external force for example on unstable surfaces like wobble boards, as a consequence of voluntary movement or in response to an unexpected perturbation or stimulus in order to maintain body's centre of mass. Balance training foreground on the maintenance of balance during visual and perturbation challenges with eyes open or closed and maintaining stability over reduced areas of support and unstable surface (18).

Hence the purpose of the study is to impart four-week weight bearing exercise program on stable platform and wobble board and find which is more effective in improving balance and functional outcome of individuals with knee osteoarthritis.

\section{MATERIALS AND METHODS}

On obtaining Institutional Review Board (IRB) approval and ethical clearance, study was conducted in Saveetha Medical College, Physiotherapy outpatient department, with study design as Quasi experimental study, sampling method as convenient sampling method, and sample size of 40 subjects divided into two groups with 20 subjects in each group. Subjects were included into study based on the inclusion criteria as willingness of individuals, both male and female subjects, age group of $40-70$ years, patient diagnosed as a case of osteoarthritis knee, bilateral knee joint involvement, radiographic evidence of grade II or grade III of Kellgren and Lawrence criteria for knee osteoarthritis and subjects were excluded if there was any secondary osteoarthritis i.e., due to trauma during last three months, due to thyroid etc., Other musculoskeletal problems associated with knee joint such as tendon or ligament tears, uncontrolled medical problems such as hypertension, angina, severe cardiomyopathy, any other locomotor or neurological disorders, open wound or skin disease, osteoporosis, bone tumours, lower limb deformities. Materials used for the study are wobble board, treatment couch, stepper platforms, measuring tape, armchair, tape, stopwatch, IFT machine, cotton and gel.

\section{Procedure}

According to inclusion and exclusion criteria prior to the study, the principal researcher explained the procedures to all the subjects and pre-treatment outcome scores for balance and disability using Timed Up and Go test (TUG test) and Knee injury and Osteoarthritis Outcome Score (KOOS) questionnaire was recorded. Using convenient sampling method, 40 patients were selected from Physiotherapy out-patient department and were allocated into two groups with 20 subjects in each group. After recruitment on eligibility, written informed consent was obtained on explaining the treatment to be given. Group A: weight bearing exercises on wobble board and Group B: weight bearing exercises on stable platform. These weightbearing exercises included 1 . Squats, 2 . Sit to stand, 3. Forward lunge, 4. Step ups, 5. Timed single leg stance and 6. Wall slides. All exercises were given 3 sessions a week for 4 weeks, each session consists of 4 sets with 6 repetitions a set and a rest interval of one minute between each set. Subjects in both the groups were given Interferential therapy with parameters- Intensity: maximum sensory, mild motor sensation, Frequency of machine: $90-130 \mathrm{~Hz}$ and Duration: 15 minutes per session. Post-treatment outcome scores for balance and disability using TUG test and KOOS questionnaire was recorded after 4 weeks.

\section{Statistical analysis}

The data was statistically analysed using Student's " $t$ " test. Paired t test was used to analyse the pre-test and post-test values within the group. Student's ' $t$ ' test was used to analyse the pre and post-test values between the groups.

Table 1: Comparison of pre-test values of KOOS outcome \& TUG test between group A \& group B

\begin{tabular}{|c|c|c|c|c|c|c|}
\hline \multirow{2}{*}{ Parameter } & \multicolumn{3}{|c|}{ Post-test values } & \multirow{2}{*}{ G value } & \multirow{2}{*}{ t value } \\
\cline { 2 - 6 } & \multicolumn{2}{|c|}{ Group A } & \multicolumn{2}{|c|}{ Group B } & & \\
\cline { 2 - 6 } & Mean & Standard deviation & Mean & Standard deviation & & \\
\hline KOOS Pain & 37.05 & 5.00 & 37.05 & 3.14 & 0.9973 & 0.0034 \\
\hline KOOS Symptoms & 30.02 & 5.35 & 31.78 & 5.89 & 0.3302 & 0.9864 \\
\hline KOOS ADLS & 30.52 & 5.67 & 31.78 & 6.75 & 0.5293 & 0.6349 \\
\hline KOOS Sports \& Recreation & 27.50 & 6.98 & 28.20 & 7.33 & 0.7588 & 0.3093 \\
\hline KOOS quality of life & 32.18 & 6.80 & 33.75 & 6.21 & 0.4532 & 0.7579 \\
\hline Timed UP and GO test & 7.535 & 1.003 & 7.820 & 0.798 & 0.3263 & 0.9943 \\
\hline
\end{tabular}

Table 2: Comparison between pre-test \& post-test values of KOOS outcome \& TUG test with in group A

\begin{tabular}{l|l|l|l|} 
Mean & Standard deviation & 'p' value & $\mathbf{t}$ value \\
\hline
\end{tabular}




\begin{tabular}{|c|c|c|c|c|c|}
\hline \multirow{2}{*}{ KOOS Pain } & Pre-test & 37.05 & 5.01 & \multirow{2}{*}{$<0.0001$} & \multirow{2}{*}{13.29} \\
\hline & Post-test & 64.44 & 7.73 & & \\
\hline \multirow{2}{*}{ KOOS Symptoms } & Pre-test & 30.02 & 5.35 & \multirow{2}{*}{$<0.0001$} & \multirow{2}{*}{21.01} \\
\hline & Post-test & 68.92 & 8.36 & & \\
\hline \multirow{2}{*}{ KOOS ADLS } & Pre-test & 30.52 & 5.67 & \multirow{2}{*}{$<0.0001$} & \multirow{2}{*}{16.88} \\
\hline & Post-test & 71.86 & 8.22 & & \\
\hline \multirow{2}{*}{ KOOS Sports \& Recreation } & Pre-test & 27.50 & 6.98 & \multirow{2}{*}{$<0.0001$} & \multirow{2}{*}{18.99} \\
\hline & Post-test & 78.25 & 10.42 & & \\
\hline \multirow{2}{*}{ KOOS Quality of Life } & Pre-test & 33.12 & 6.44 & \multirow{2}{*}{$<0.0001$} & \multirow{2}{*}{13.47} \\
\hline & Post-test & 72.18 & 9.61 & & \\
\hline \multirow{2}{*}{ Timed UP and GO Test } & Pre-test & 7.53 & 1.00 & \multirow{2}{*}{$<0.0001$} & \multirow{2}{*}{11.99} \\
\hline & Post-test & 5.34 & 0.71 & & \\
\hline
\end{tabular}

Table 3: Comparison between pre-test \& post-test values of KOOS Outcome \& TUG test with in group B

\begin{tabular}{|c|c|c|c|c|c|}
\hline \multicolumn{2}{|l|}{ GROUP B } & Mean & Standard deviation & 'p' value & t value \\
\hline \multirow{2}{*}{ KOOS Pain } & Pre-test & 37.05 & 3.14 & \multirow{2}{*}{$<0.0001$} & \multirow{2}{*}{5.83} \\
\hline & Post-test & 50.69 & 10.34 & & \\
\hline \multirow{2}{*}{ KOOS Symptoms } & Pre-test & 31.78 & 5.89 & \multirow{2}{*}{$<0.0001$} & \multirow{2}{*}{9.43} \\
\hline & Post-test & 54.64 & 9.97 & & \\
\hline \multirow{2}{*}{ KOOS ADLS } & Pre-test & 31.78 & 6.75 & \multirow{2}{*}{$<0.0001$} & \multirow{2}{*}{15.67} \\
\hline & Post-test & 58.68 & 5.60 & & \\
\hline \multirow{2}{*}{ KOOS Sports \& Recreation } & Pre-test & 27.75 & 6.78 & \multirow{2}{*}{$<0.0001$} & \multirow{2}{*}{14.10} \\
\hline & Post-test & 63.00 & 9.79 & & \\
\hline \multirow{2}{*}{ KOOS Quality of Life } & Pre-test & 33.75 & 6.21 & \multirow{2}{*}{$<0.0001$} & \multirow{2}{*}{9.27} \\
\hline & Post-test & 52.18 & 9.57 & & \\
\hline \multirow{2}{*}{ Timed UP and GO Test } & Pre-test & 7.82 & 0.79 & \multirow{2}{*}{$<0.0001$} & \multirow{2}{*}{9.90} \\
\hline & Post-test & 6.78 & 0.76 & & \\
\hline
\end{tabular}

Table 4: Comparison of post-test values of KOOS outcome \& TUG test between group A \& group B

\begin{tabular}{|c|c|c|c|c|c|c|}
\hline \multirow{2}{*}{ Parameter } & \multicolumn{3}{|c|}{ Post-test values } & \multirow{3}{*}{ G value } & \multirow{2}{*}{ t value } \\
\cline { 2 - 6 } & \multicolumn{3}{|c|}{ Group A } & \multicolumn{2}{|c|}{ Group B } & \\
\cline { 2 - 6 } & Mean & Standard deviation & Mean & Standard deviation & & \\
\hline KOOS Pain & 64.44 & 7.73 & 50.69 & 10.34 & $<0.0001$ & 4.76 \\
\hline KOOS Symptoms & 68.92 & 8.36 & 54.64 & 9.97 & $<0.0001$ & 4.90 \\
\hline KOOS ADLS & 71.86 & 8.22 & 58.68 & 5.60 & $<0.0001$ & 5.92 \\
\hline KOOS Sports \& Recreation & 78.25 & 10.42 & 63.00 & 9.79 & $<0.0001$ & 4.77 \\
\hline KOOS quality of life & 72.18 & 9.61 & 52.18 & 9.57 & $<0.0001$ & 6.59 \\
\hline Timed UP and GO test & 5.34 & 0.71 & 6.78 & 0.76 & $<0.0001$ & 6.19 \\
\hline
\end{tabular}

\section{RESULTS}

From the statistical analysis, the quantitative data revealed statistically significant difference between Group A and Group B and within the groups.

Table1 showed pre-test mean, standard deviation, significance (p) values of both the groups. On analysis and comparison, it was observed that there was homogeneous distribution of subjects in both the groups.

Table 2 showed pre and post-test mean and standard deviation along with significance (p) value of Group A. The mean values showed that there is a significant improvement in all the 5 components of KOOS questionnaire and TUG test following intervention. The significance (p) value of all the 5 components of KOOS questionnaire and TUG test was found to be extremely significant $(\mathrm{p}<0.0001)$.

Table 3 showed pre and post-test mean and standard deviation along with significance (p) value of Group B. The mean values showed that there is little improvement in all the 5 components of KOOS questionnaire and TUG test following intervention. The significance (p) value of all the 5 components of KOOS questionnaire and TUG test was found to be extremely significant $(\mathrm{p}<0.0001)$. 
Table 4 showed post-test mean, standard deviation, significance $(p)$ values of both groups. On analysis and comparison, the post-test mean values of Group A individuals showed significant improvement than Group B individuals.

\section{DISCUSSION}

Knee osteoarthritis generally defined by narrowing of the joint space, is a prevalent condition that is often associated with debilitating pain (19). The prevalence of OA increases with age and generally affects women more frequently those men $(2,4)$. Lack of education, corpulence and reduced muscular strength are presenting symptoms in symptomatic disease and subsequent disability (20). All of the above coincides with this study.

Primary osteoarthritis is a common disorder of the elderly, and individuals are often asymptomatic. On the basis of the radiographic criteria for osteoarthritis, $50 \%$ of the adults older than 65 years are affected by the disease (21). Clinically OA is characterised by pain during weight bearing, tenderness and limitation of knee movement, crepitus, occasional effusion and variable degrees of local inflammation $(1,7,8)$, along with reports of subjects suffering from impaired balance and disability.

Subjects with OA knee have impairment of proprioception within the joint or weakness in the quadriceps muscles as compared with those without OA knee. These impairments associated with the disease may explain poorer balances within these individuals (22). Previous evidences suggest the possibility that quadriceps weakness is the primary risk factor for knee pain, disability, balance and progression of joint damage in individuals with osteoarthritis of knee (23).

In this study, the key focus area was on functional outcome and balance in knee osteoarthritic individuals. The primary goal was to determine the effect of weight bearing exercises on stable platform versus wobble board in knee osteoarthritic individuals using KOOS questionnaire (24) and Timed Up and Go test (25). The results of the study showed significant improvement in Group A, in which weight bearing exercises were performed on wobble board, in terms of balance and functional outcome.

Individuals included in this study had similar baseline values of all dependent variables selected, suggesting that both groups had homogenous distribution of individuals. The analysis of the data collected revealed that subjects in both the groups significantly improved in terms of functional outcome and balance as depicted on KOOS questionnaire and TUG test scores compared to the baseline values. The comparative results of this study depict that weight bearing exercises on wobble board for knee osteoarthritic individuals is more effective in improving balance and functional outcome than weight bearing exercises on stable platform.

As suggested by Kim burton in his study, trunk muscle activity induced by wobble board usage in balance training improve proprioceptive response from articular mechanoreceptors following injury thereby improving balance (3).

Weight bearing exercises were found to increase coordination between muscle groups and improve response to sensorial information. Each exercise is found to elicit automatic and reflexive muscular stabilization demanding the individual to maintain postural control under a variety of situations (15).

From previous studies done weight bearing exercises are also found to improve muscle strength that is supposed to be one of the causes of OA knee, functional status and balance of individuals with knee osteoarthritis. In another study, it was clearly indicated that wobble board training and stability trainer both can be inculcated as a part of rehabilitation in Knee OA patients depending on the variables we want to focus on and improve (3).

Therefore, the present study was done to study the effect of weight bearing exercises on wobble board, which proved to be effective in improving functional outcome and balance in knee osteoarthritic individuals.

Another previous study suggests that, Application of Interferential therapy to the painful knee with amplitude modulated and low frequencies is found to reach deep muscles and nerves, stimulate voluntary muscles, promotes an increase peripheral blood flow and reduces pain (13). This was co-related with present study as individuals reported of reduction in pain level after giving Interferential therapy.

So, with reference to previous literature subjects in this study were benefited in terms of improvement in balance and functional outcome that were obtained by performing weight bearing exercises on wobble board along with Interferential therapy that helps in performing exercises with minimum or no pain. So, this study clearly indicates that weight bearing exercises on wobble board are more effective and can be inculcated in rehabilitation of OA knee individuals to improve functional outcome and balance.

\section{CONCLUSION}

It is concluded in the present study that there was a statistically significant difference in balance and functional outcome with weight bearing exercises on wobble board as compared to weight bearing exercises on stable platform in subjects with knee osteoarthritis. Although both the groups were found to be effective in improving balance and functional outcome, however comparative results depicted that the subjects with weight bearing exercises done on wobble board showed more improvement. Hence it is 
concluded that weight bearing exercises on wobble board might be a better therapeutic intervention as compared to weight bearing exercises on stable platform for treating subjects with OA knee.

\section{CONFLICT OF INTEREST}

All contributing authors declare no conflicts of interest.

\section{REFERENCES}

1. Metgud, S., Putti, B. B. Effect of proprioceptive exercises in Osteoarthritic and replaced knees. International Journal of Physiotherapy and Research. 2015; 3(6): 1294-1300.

2. Mei -Hwa Jan., Chien- Ho Lin., Yeong-Fwu Lin. Effects of weight-bearing versus non weight-bearing exercise on function, walking speed, and position sense in participants with knee Osteoarthritis: a randomized controlled trial. Arch Phys Med Rehabil. 2009; 90: 897-904.

3. Grover, S., Khanduja, K. Effect of training with stability trainer vs wobble board training on proprioception, balance and function in patients with knee osteoarthritis. International journal for scientific research and development. 2017; 5(7): 292-295.

4. Silva, A., Serrao, P. R. M. S., Driusso, P., Mattiello, S. M. The effects of therapeutic exercise on the balance of women with knee Osteoarthritis: a systematic review. Rev Bras Fisioter. 2012; 16(1): 1-9.

5. Mahajan, A., Verma, S., Tandon, V. Osteoarthritis. Journal of the association of Physicians of India. 2005; 53: 634-641.

6. Bhagat, P., Jagtap, V., Devi, P. T. Effect of circuit training in osteoarthritis of knee. Asian Journal of Pharmaceutical and Clinical Research. 2017; 10(7): 333-335.

7. Gangaram, J. S., Stephen B. A. A study on effect of proprioceptive training in patients with osteoarthritis knee wearing elastic bandages. The Indian Journal of Occupational Therapy. 2009; XLI (2): 37-42.

8. Pradip Kumar, G., Ray, D., Chatterjee, B., Acharya, S., Adhikary, S., Anindita De. Comparative study of the effectiveness between balancing exercises and strengthening exercises with common use of TENS to improve functional ability in Osteoarthritis involving knee joint. International Archives of Integrated Medicine.2015; 2(10): 1-17.

9. Ju, S. B., Park, G. D., Kim, S. S. Effects of proprioceptive circuit exercise on knee joint pain and muscle function in patients with knee osteoarthritis. J. Phys. Ther. Sci. 2015 ;27: 2439-2441.

10. Sisto S. A, Malanga, G. Osteoarthritis and therapeutic exercise. Am J Phys Med Rehabil. 2006; 85: S69-S78.

11. Dhebar, F. Interventions for increasing balance \& confidence in older adults: A Review International Journal of Physiotherapy and Research. 2014; 2(4): 631-636.

12. Nehal, S., Megha, S. Role of interferential therapy in Osteoarthritis knee a narrative review. Journal of Evidence Based Physiotherapy and Research. 2017; 1(1): 12-16.

13. Archana, B., Nilima, B. Comparison of effects of interferential therapy (IFT) and combination therapy (IFT+ ultrasound therapy) on pain, range of motion and function in patients with osteoarthritis of knee: A hypothesis. Journal Medical Thesis. 2015; 3(2): 3-7.

14. Pournima, P., Rajkumari K. P., Ujwal, L. Y. Effects of TENS vs IFT in patients on pain and function on osteoarthritis of knee: A randomized control trial. International Journal of Yoga, Physiotherapy and Physical Function. 2018; 3(2): 83-86.

15. Ahmed, A. F. Effects of Sensorimotor training on balance in elderly patients with knee Osteoarthritis. Journal of Advanced Research. 2011; 2: 305-311.

16. Winter, D. A., Human balance and posture control during standing and walking. Gait and Posture. 1995; 3: 193-214.

17. Prakash, J., Irshad, A., Sonal, K., Ali, K., Shalini, V., Kumar, T. Proprioception: An evidence based narrative review. Research and Investigations in Sports Medicine. 2017; 1(2): 1-5.

18. Silva, K. N. G., Mizusaki, I, A., Almeida, G. J. M., Atallah, A. N., Peccin, M. S., Moca, F., et al., Balance training (proprioceptive training) for patients with rheumatoid arthritis (Review). Cochrane Database of Systematic Reviews. 2010; 5: 1-19.

19. Mazzuca, S. A., Brandt, K. D. Is knee radiography useful for studying the efficacy of a disease- modifying osteoarthritis drug in humans? Rheumatic disease clinics of North America. 2003; 29: 819-830.

20. Peter, W. Lementowski, S., Zelicof, B. Obesity and Osteoarthritis. The American journal of Orthopedics. 2008; 37(3): 148-151.

21. Roberts, J., Thomas, A. B. Prevalence of Osteoarthritis in adults by age, sex, race, and geographic area. 1960-62; 11(15): 1-27.

22. Jeong, P. H., Ko, S., Hong, H., Eunjae, O., Lee, J. I. Factors related to standing balance in patients with knee osteoarthritis. Ann Rehabil Med. 2013; 37(3): 373-378.

23. Charles, S., Kenneth, D. B., Douglas, K., Steven, M. H., Ethnan, M., Braunstein, B. P. K., et al., Quadriceps weakness and osteoarthritis of the knee. Annals of Internal Medicine. 1997; 127(2): 97-104.

24. Roos, E. M., Roos, H. P., Lohmander, L. S., Ekdahl, C., Beynnon, B. D. Knee injury and Osteoarthritis Outcome Score (KOOS) development of a self-administered outcome measure. J Orthop Sports Phys Ther. 1998; 28(2): 88-96.

25. Bohannon, R. W. Reference values for the Timed Up and Go Test: A descriptive meta- analysis. Journal of Geriatric Physical Therapy. 2006; 87(2): 64-68. 